\title{
El DESARROLLO DE NUEVOS PRODUCTOS A LA LUZ Y A LA SOMBRA DE LAS CAPACIDADES DINÁMICAS*
}

\author{
Ana María Ortega Álvarez ${ }^{* *}$ \\ Teresa García Merino ${ }^{* * *}$ \\ María Valle Santos Álvarez ${ }^{* * * *}$
}

El presente artículo es resultado de una investigación realizada por el Departamento de Organización de Empresas de la Universidad de Valladolid, España, titulado "El desarrollo de nuevos productos a la luz y sombra de las capacidades dinámicas", llevada a cabo desde el 12-06-2011 hasta el 10-12-2012. El artículo se recibió el 12-06-2012 y se aprobó el 16-10-2012.

** Licenciada en Ciencias Económicas y Empresariales, Universidad de Valladolid, Valladolid, España, 1993. Profesora titular, Universidad de Valladolid, Valladolid, España. Miembro del grupo de investigación Organización de Empresas. Correo electrónico: anao@eade.uva.es

*** Ph.D. en Ciencias Económicas y Empresariales, Universidad de Valladolid, Valladolid, España, 1995. Profesora titular, Universidad de Valladolid, Valladolid, España. Miembro del grupo de investigación Organización de Empresas. Correo electrónico: temerino@eco.uva.es

**** Ph.D. en Ciencias Económicas y Empresariales, Universidad de Valladolid, Valladolid, España, 1997. Profesora titular, Universidad de Valladolid, Valladolid, España. Miembro del grupo de investigación Organización de Empresas. Correo electrónico: mvalle@eco.uva.eso 
El desarrollo de nuevos productos a la luz y a la sombra de las capacidades dinámicas

\section{RESUMEN}

El objetivo de esta investigación es analizar el proceso de desarrollo de nuevos productos (DNP) como mecanismo de renovación organizativa. Para ello se revisa la literatura sobre DNP desde la óptica de las capacidades dinámicas, que cubre el análisis del entorno y la redefinición del dominio de actividad, pasando por la reconfiguración de las capacidades operativas. De la revisión de la literatura se constata que los procesos de DNP se pueden reinterpretar como capacidades dinámicas y, por ello, pueden servir como mecanismos de renovación organizativa. Sin embargo, a partir de reconocer las limitaciones de esta concepción y la necesidad de recuperar el concepto de capacidad organizativa, se considera que la dinamización de estas capacidades representa un proceso de renovación más abierto e inspirado en los retos del entorno.

Palabras clave: desarrollo de nuevos productos, capacidades dinámicas, capacidades organizativas, renovación organizativa

Clasificación JEL: L20, M10

\section{New product development in light (and shadow) of dynamic capabilities}

\begin{abstract}
The objective of this research is to analyze the process of New Product Development (NPD) as a mechanism for organizational renewal. To this end, the literature is reviewed from the perspective of dynamic capabilities, which comprise both environment analysis and activity domain redefinition, via the reconfiguration of operational capabilities. A review of the literature finds that NPD processes can be reinterpreted as dynamic capabilities and therefore can serve as mechanisms for organizational renewal. However, the literature also recognizes the limitations of this view and the interest to retrieve the concept of organizational capability. The process of capability dynamization represents a broader renewal process, inspired on the challenges of the environment.
\end{abstract}

Keywords: new product development, dynamic capabilities, organizational capabilities, organizational renewal

JEL Classification: L20, M10

\section{O desenvolvimento de novos produtos a luz e a sombra das capacidades dinâmicas}

\section{RESUMO}

O objetivo desta pesquisa é analisar o processo de desenvolvimento de novos produtos (DNP) como mecanismo de renovação organizacional. Para isso, é revista a literatura sobre DNP, do ponto de vista das capacidades dinâmicas, que abrange a análise do entorno e a redefinição do domínio de atividade, passando pela reconfiguração das capacidades operacionais. Com a revisão da literatura, constata-se que os processos de DNP podem ser reinterpretados como capacidades dinâmicas e, por isso, podem servir como mecanismos de renovação organizacional. Mas, a partir de reconhecer as limitações desta concepção, e a necessidade de recuperar o conceito de capacidade organizacional, considera-se que a dinamização destas capacidades representa um processo de renovação mais aberto e inspirado nos desafios do entorno.

Palavras chave: Desenvolvimento de novos produtos, capacidades dinâmicas, capacidades organizacionais, renovação organizacional 


\section{Introducción}

El desarrollo de nuevos productos (en adelante DNP) es, sin duda, uno de los procesos organizativos más ampliamente estudiados y del que, cada vez más, se destaca el importante papel que desempeña en la capacidad adaptativa y en la renovación de la organización. Precisamente, en lo relativo a la necesidad de renovación organizativa, hemos asistido en los últimos años al desarrollo y consolidación, en el campo de la dirección estratégica, del concepto de capacidades dinámicas. En efecto, la importancia que las capacidades dinámicas tienen de cara a la supervivencia y éxito de la empresa es sobradamente reconocida hoy en día, y prueba de ello es la creciente atención que se les ha venido dedicando en la literatura. No obstante, la noción de capacidades dinámicas mantiene algunos de los rasgos de abstracción e imprecisión que se le han atribuido desde que se acuñó el término, lo que ha llevado a algunos autores a señalar la necesidad de identificar tales capacidades con determinados procesos organizativos. Tomando esta idea como punto de partida, nuestro trabajo se centra en analizar si el DNP puede ser considerado como uno de los procesos que constituyen capacidades dinámicas de la organización. El cumplimiento de este objetivo nos llevará a revisar, en primer lugar, el propio concepto de capacidad dinámica. Asimismo, con el fin de ofrecer una visión más completa del proceso de DNP, expondremos también las diferentes opciones que puede presentar.

Más concretamente, los apartados en los que se estructura el presente trabajo son: en primer lugar y tras la introducción, una breve revisión de los fundamentos teóricos del enfoque de capacidades dinámicas, señalando también algunos de sus aspectos más controvertidos. El tercer epígrafe se centra en el DNP, empezando por delimitar dicho proceso, para a continuación ponerlo en relación con la noción de capacidad dinámica presentada. Se analiza de esta manera si el DNP reúne los rasgos como para ser considerado una de tales capacidades, y se cierra el epígrafe con la revisión, ya más pormenorizada, de las distintas opciones que desde esta óptica puede presentar. Finalizamos el trabajo con las conclusiones más relevantes del mismo.

\section{Las capacidades dinámicas: qué sabemos}

El debate actual en el campo de la dirección estratégica admite ampliamente la noción de capacidades dinámicas, en aras de superar las potenciales rigideces de la construcción de capacidades organizativas (Schreyögg y Kliesch-Eberl, 2007). El concepto de capacidades dinámicas, si bien había aparecido con anterioridad ${ }^{1}$, se generaliza a partir del trabajo de Teece et ál. (1997), centrándose en los mecanismos mediante los cuales las empresas acumulan, reconfiguran y despliegan nuevas capacidades, y constituye una llamada de atención sobre la necesidad de renovar las capacidades para mantener la adaptación con el entorno.

1 La definición de capacidades dinámicas es similar a las ofrecidas por otros autores que habían utilizado parecidos términos: combinative capabilities de Kogut y Zander (1992), architectural competence de Henderson y Cockburn (1994) o capabilities de Amit y Schomaker (1993). 
En efecto, las capacidades dinámicas se definen como la habilidad de la empresa para integrar, construir y reconfigurar las competencias y enfrentarse al entorno cambiante (Teece et ál., 1997, p. 515). Mientras el concepto de capacidades involucra la coordinación de múltiples actividades y actores organizativos en la búsqueda de un objetivo específico, como la adaptación o el crecimiento (Helfat y Peteraf, 2003), el término dinámicas alude a la necesidad de renovación.

En un esfuerzo por comprender la verdadera naturaleza de las capacidades dinámicas, diversos autores señalan la necesidad de empezar estableciendo una distinción entre los diferentes tipos de procesos y rutinas existentes en las empresas. Aunque existen diferentes conceptualizaciones en este sentido, son numerosos los investigadores que afirman que la característica distintiva de las capacidades dinámicas radica en su papel de reconfiguración de las capacidades operativas (Helfat et ál., 2007; Zahra et ál., 2006; Zollo y Winter, 2002).

Henderson y Cockburn (1994) y Collis (1994) son algunos de los primeros autores que recogen en sus trabajos dicha distinción. Collis diferencia entre una primera categoría de capacidades, que refleja la habilidad de realizar las actividades funcionales básicas de la empresa, y una segunda categoría, la cual tiene que ver con la mejora dinámica de las actividades de la empresa. Las capacidades dinámicas gobiernan la tasa de cambio de las capacidades ordinarias.

Zollo y Winter (2002) y Winter (2003) también diferencian entre capacidades operati- vas (zero-order) y dinámicas (first-order). Las capacidades dinámicas se dedican a la modificación de las capacidades operativas y llevan, por ejemplo, a cambios en los productos o en los procesos productivos. Mientras las capacidades operativas se refieren a “how you earn your living”, las capacidades dinámicas se ocupan de "how you change your operational routines" (Helfat y Peteraf, 2003; Winter, 2003).

Cepeda y Vera (2007) perfilan con exactitud la distinción entre capacidades dinámicas y operativas al señalar que el input de las capacidades dinámicas es la configuración inicial de recursos y rutinas operativas, y que las capacidades dinámicas constituyen un proceso de transformación de éstos, siendo su output una nueva configuración de recursos y rutinas operativas.

La distinción entre ambos tipos de capacidades, adoptada cada vez más en la literatura, está contribuyendo a eliminar el matiz tautológico tradicionalmente asociado a las capacidades dinámicas, que se ha debido sobre todo a la incorporación en ellas del concepto de ventaja competitiva (Priem y Butler, 2001). Las definiciones más recientes que diferencian entre capacidades operativas y dinámicas argumentan que la ventaja competitiva proviene de las nuevas configuraciones de recursos y capacidades operativas conseguidas, y no de las capacidades dinámicas per se (Eisenhardt y Martin, 2000; Makadok, 2001). Las capacidades dinámicas contribuyen indirectamente a los resultados, mediante su impacto sobre las capacidades operativas (Helfat y Peteraf, 2003), y alteran la configuración de recursos, rutinas y competencias, 
lo cual a su vez afecta a los resultados organizativos (Zott, 2003).

Al margen de contribuir a afianzar la distinción entre capacidades operativas y dinámicas, la literatura reciente en este campo ha avanzado también en lo relativo a comprender mejor el propio funcionamiento de las capacidades dinámicas, tratando de desentrañar sus mecanismos internos.

En este sentido, Lavie (2006) ofrece un modelo de reconfiguración de las capacidades empresariales que se desarrolla en dos etapas y en el cual se pueden utilizar tres mecanismos. Si bien no introduce el término capacidades operativas, el autor se refiere claramente a ellas y las toma como unidad de análisis. Las dos etapas del proceso de reconfiguración son: una primera fase en la cual los decisores de la empresa reúnen información sobre determinado cambio ocurrido en el entorno y analizan sus implicaciones, lo que puede llevarlos a redefinir la configuración de capacidades maximizadora de valor, y una segunda fase, en la cual actúan sobre la base de capacidades con el fin de ajustarla a la nueva configuración.

Los tres mecanismos que la empresa puede utilizar para llevar a cabo la reconfiguración de las capacidades son la sustitución, la evolución y la transformación. El mecanismo de sustitución implica que la configuración de capacidades que posee la empresa es invariante o altamente inerte ante el cambio. En contraste, el mecanismo de evolución parte de que el cambio externo desencadena procesos de reconfiguración de las rutinas que componen las capacidades existentes siguiendo una senda de dependencia con respecto al pasado. Por su parte, con el mecanismo de transformación de capacidades, algunas rutinas son modificadas, otras son eliminadas y algunas nuevas rutinas son incorporadas. Esto lo convierte en un mecanismo intermedio, que da como resultado una nueva configuración de capacidades, la cual conjuga conocimiento y habilidades existentes (como en la evolución) con conocimiento y habilidades nuevos (como en el mecanismo de sustitución). Lavie (2006) considera que el enfoque de capacidades dinámicas se centra fundamentalmente en el segundo mecanismo, entendiendo que la evolución se produce mediante un proceso de prueba y error de las rutinas que componen las capacidades operativas.

Otra interesante aportación al objetivo de profundizar en el funcionamiento interno de las capacidades dinámicas la encontramos en Teece $(2007,2009)$. Este autor estudia los fundamentos directivos de las capacidades dinámicas, es decir, trata de descubrir cuáles son las habilidades, procesos, procedimientos, estructuras organizativas, reglas de decisión y disciplinas que hacen que la empresa consiga crear, desplegar y proteger su base de recursos y competencias.

Las capacidades dinámicas son desagregadas en tres componentes: la capacidad de identificar oportunidades y amenazas, la capacidad de aprovechar oportunidades y la capacidad propiamente dicha de reconfigurar la base de recursos y capacidades. Las habilidades y fundamentos directivos son agrupados en relación con dichos componentes. 
Es de destacar el paralelismo entre las aportaciones de Lavie (2006) y Teece (2007, 2009), ambas recogidas posteriormente por un buen número de investigadores, en lo relativo al proceso de desarrollo de una capacidad dinámica: ambos coinciden en señalar la detección de los estímulos del entorno como punto de partida que proporciona el conocimiento necesario para emprender un proceso de transformación que culmine en una configuración de recursos y capacidades más ventajosa. Como reconocen Helfat et ál. (2007), podemos afirmar que el concepto de capacidad dinámica ha sido ampliado gradualmente hasta incluir la capacidad de identificar la necesidad u oportunidad de cambio, de formular una respuesta y de implementar cursos de acción. Así, aunque en un principio las capacidades dinámicas se asociaban fundamentalmente con procesos de transformación de las capacidades operativas, con el tiempo se les están incorporando otros roles, como la habilidad de toma de decisiones o aquella de detección de estímulos externos (Barreto, 2010).

Podemos sintetizar las aportaciones anteriores en una propuesta integradora que defina las capacidades dinámicas como procesos de reconfiguración de capacidades operativas sustentados en procesos de análisis del entorno. La reconfiguración del dominio de actividades de la empresa constituye el punto final en el que desembocan dichos procesos.

Ahora bien, el mismo concepto de capacidades dinámicas, que emerge como elemento de ventaja frente a competidores, puede convertirse también en un elemento de rigidez que dificulte la propia renovación de la em- presa. En efecto, en determinados momentos la empresa se compromete de forma casi irreversible con ciertas áreas de competencias (Teece et ál., 1997), que se consolidan así como sus capacidades centrales y al tiempo se convierten en límite para futuros procesos de cambio (Leonard-Barton, 1992).

Esta disyuntiva nos conduce a la controversia existente en la literatura en relación con el concepto de capacidad dinámica y el proceso de dinamización de las capacidades organizativas (Schreyögg y Kliesch-Eberl, 2007; Dávila, 2012). Dicha controversia subraya la incoherencia que se produce entre el concepto de capacidad dinámica — caracterizada por el dinamismo y la flexibilidad - y el de capacidad organizativa — con patrones de acción repetitivos y, simultáneamente, función de reconfiguración-. En consecuencia y con el objeto de preservar el concepto de capacidad organizativa, Schreyögg y Kliesch-Eberl (2007) proponen atender a la distinción entre construcción y práctica de la capacidad, por un lado, y su dinamización, por el otro. Distinción a la que se denomina proceso dual de dinamización de las capacidades. La dinamización se concibe así como un proceso abierto, libre de las ataduras de lo rutinario y lo preestablecido, que deviene de la reflexión acerca de la idoneidad de la capacidad y la dinámica del entorno (Dávila, 2012). Se pone hincapié, además, en la necesaria separación entre la práctica de la capacidad y la reflexión sobre dicha práctica, que deberá ser realizada desde la perspectiva de observadores situados en un nivel superior.

La consideración de esta crítica al concepto de capacidades dinámicas nos lleva a re- 
flexionar sobre hasta qué punto las capacidades organizativas admiten la posibilidad de dinamización y de evolución, adaptándose a los cambios del entorno. Nuestra posición al respecto admite la noción de capacidad dinámica, en cuanto que la evolución para mantener la adaptación a las condiciones del entorno puede, con frecuencia, presentar una orientación clara hacia ámbitos cercanos a la actual base de capacidades de la empresa. Una transformación más profunda y alejada del actual repertorio de capacidades, seguramente requiera un proceso de observación y reflexión realizado a una determinada distancia del desarrollo cotidiano de dichas capacidades, lo que nos aproximaría más al concepto de capacidades organizativas y proceso dual de dinamización.

\section{El proceso de desarrollo de nuevos productos como capacidad dinámica}

Tras revisar las principales aportaciones del enfoque de capacidades dinámicas, nuestra atención se dirige ahora hacia el proceso de DNP. Empezaremos por delimitar dicho proceso y a continuación trataremos de ponerlo en relación con la noción de capacidad dinámica, anteriormente presentada. Finalmente, realizaremos una revisión, ya más pormenorizada, de las distintas opciones que puede presentar el DNP.

\section{El proceso de desarrollo de nuevos productos}

El desarrollo de un nuevo producto es uno de los procesos organizativos más complejos, dado que requiere la capacidad de obtener, procesar e interpretar gran cantidad de información, para desarrollar ideas de producto y evaluar sus límites técnicos, posibilidades de fabricación y viabilidad económica (Gomes et ál., 2003).

La literatura sobre desarrollo de productos ha crecido significativamente, si bien se puede calificar de variada y fragmentada (Brown y Eisenhardt, 1995). Constituye una de las dos grandes ramas en las que se puede dividir la investigación en innovación. La primera rama, de base económica, estudia los patrones de innovación en distintas industrias y la evolución de las tecnologías. El proceso de desarrollo de productos constituye en buena medida una caja negra. La segunda rama, la de desarrollo de productos, trata de abrir la caja negra, explicando cómo se generan nuevos productos dentro de las empresas y, de forma especial, en qué consiste este proceso y en qué estructuras se apoya.

El desarrollo de productos puede ser denominado también innovación de productos y es "una práctica que comprende el enlace creativo de posibilidades comerciales y tecnológicas en un paquete general de atributos" (Dougherty, 1992, p. 78). El desarrollo de un nuevo producto es, por tanto, un proceso organizativo en el que se vinculan tecnología y clientes. Desde el lado de la demanda, las necesidades de los clientes los llevan a pedir ciertos beneficios a los productos. Desde la óptica de la oferta, las tecnologías de la empresa la capacitan para proporcionar tales beneficios a través de los atributos de sus productos. 
Las dos tareas clave incluidas en el DNP son, por tanto, elaborar el producto y venderlo a ciertos consumidores, por lo que dicho proceso requiere que la empresa posea dos tipos fundamentales de capacidades, que por su naturaleza podemos considerar como claros ejemplos de capacidades operativas: las tecnológicas y las relacionadas con los clientes o capacidades de mercado (Danneels, 2002). Capacidad tecnológica es aquella que otorga a la empresa la habilidad para diseñar y fabricar un producto con ciertas características y se genera a partir de recursos como la planta de producción y su equipamiento, el know how de diseño y fabricación o las herramientas de control de calidad. Por su parte, capacidad de mercado es aquella que otorga a la firma la habilidad para servir a ciertos consumidores e involucra recursos tales como el conocimiento de las necesidades de los clientes, los canales de distribución y de comunicación utilizados, la reputación y la imagen de marca.

Como hemos señalado anteriormente, buena parte de la literatura sobre desarrollo de productos se ha centrado en analizar el propio proceso de desarrollo, cuestión en la que nos detenemos brevemente a continuación. Brown y Eisenhardt (1995) destacan como factores determinantes del éxito de un nuevo producto, la combinación de un producto atractivo, un mercado munificente y un eficiente proceso de desarrollo. Montoya-Weiss y Calantone (1994) señalan que de los cuatro factores más influyentes en el éxito o fracaso del nuevo producto, tres de ellos tienen que ver con su proceso de desarrollo: eficiencia en actividades técnicas y en actividades comerciales y protocolo seguido.
Entre los factores que afectan el buen desarrollo del proceso, en los trabajos de investigación revisados se señala, en primer lugar, la importancia del equipo responsable del proyecto y de que tenga carácter interfuncional. De esta forma, se incrementa la cantidad y variedad de información disponible, lo que permite abordar el DNP más rápida y completamente. Junto con la interdisciplinariedad, Brown y Eisenhardt (1995) destacan la importancia de contar con una comunicación fluida, una forma adecuada de organizar el trabajo y la utilización de distintas estrategias de resolución de problemas ante diferentes circunstancias. Así, en mercados estables, para llevar a cabo el DNP es apropiado un proceso racional y bien planificado, mientras que en mercados muy cambiantes la sustitución de la planificación por el aprendizaje conduce a mejores resultados.

Si bien la presencia de equipos interfuncionales aparece como la pieza clave para el desarrollo eficiente del DNP, otros factores de gran importancia son el líder del proyecto y la alta dirección, quienes además de contribuir al mismo a través de su función de liderazgo y de apoyo y provisión de recursos, respectivamente, son claves en la creación del concepto de producto.

Los factores que acabamos de señalar, si bien en cierto modo se pueden considerar como rasgos generales en este tipo de procesos, no siempre se manifiestan de la misma manera. Por este motivo, encontramos en la literatura diversas tipologías de procesos de DNP, entre ellas la que distingue entre procesos de tipo tradicional, flexible y basados en el conocimiento (Nonaka y Takeuchi, 1995). Otra de 
las tipologías más extendidas es la que diferencia entre procesos de primera, segunda y tercera generación (Cooper, 1994). El tipo de proceso seguido está condicionado por ciertos rasgos organizativos: el interés por la innovación, la descentralización y la experiencia previa en DNP favorecen el desarrollo de procesos con alta orientación al mercado (segunda y tercera generación).

Salvato (2009) defiende que sea cual sea el tipo de proceso seguido por la empresa para desarrollar nuevos productos, éste evolucionará a lo largo del tiempo. Es probable que exista un "tipo ideal" de DNP, con una secuencia de actividades bien definida, pero difícilmente alguno de los proyectos lo seguirá de forma exacta. Las aportaciones de los participantes provocarán variaciones sobre el tipo ideal, y si la dirección las considera útiles, las incorporará al proceso con el fin de mejorarlo. A modo de ejemplo, en la empresa estudiada por este autor, la italiana Alessi, el proceso de DNP se descompone en dieciocho actividades, coincidentes en buena medida con las señaladas en otras investigaciones, en las que el número de actividades oscila entre tres y treinta. En efecto, las diferencias entre listados son más bien numéricas o de nomenclatura, pues las tareas varían poco de unos autores a otros (Varela y Benito, 2005).

\section{El desarrollo de nuevos productos a la luz de las capacidades dinámicas}

Una vez delimitados, por un lado, la noción de capacidades dinámicas, $\mathrm{y}$, por el otro, el proceso de DNP, es el momento de poner en relación ambas cuestiones, planteándonos si el DNP, por su naturaleza y particulares carac- terísticas, reúne las condiciones necesarias como para ser considerado una capacidad dinámica.

Para ello, debemos partir de la noción de DNP que introducíamos anteriormente, y que lo conceptualizaba como proceso que mantiene una estrecha relación con las competencias o capacidades de la empresa (Danneels, 2002), en el cual se manejan principalmente dos tipos de capacidades de nivel operativo: las tecnológicas y las comerciales. El DNP se sirve de estas capacidades y las explota de determinada manera, como ha sido puesto de manifiesto reiteradamente por la literatura centrada en desarrollo de productos. Ahora bien, lo que debemos preguntarnos ahora es si, además de utilizarlas, el DNP sirve para desarrollar las capacidades de la empresa, reconfigurar su base inicial de capacidades tecnológicas y comerciales y redefinir su dominio, con el fin de responder a los requerimientos del entorno y aprovechar las oportunidades seleccionadas.

Empezamos por abordar una cuestión que en los últimos años ha ido centrando el interés de cada vez un mayor número de investigadores, coincidiendo sin duda con el progresivo desarrollo del enfoque de capacidades dinámicas: la idea de que la fuente de ventaja competitiva a largo plazo de una empresa es cada vez más dependiente de su habilidad para construir, coordinar e integrar conjuntos únicos de recursos tecnológicos y comerciales a través del DNP. Idea que es compartida por cada vez más autores (Perks, 2004).

Presentamos a continuación algunas de las principales aportaciones al respecto, siguien- 
do un orden cronológico que refleja la evolución seguida. La síntesis de tales aportaciones puede verse en el cuadro 1. Mientras en los primeros trabajos se reconoce la utilidad del DNP para mantener a la organización convenientemente renovada, pero sin mencionar aún la noción de capacidad dinámica, podemos observar cómo ésta se va asentando de manera progresiva, utilizando cada vez más la distinción entre capacidades dinámicas y operativas para conceptualizarla.

Tomamos como punto de partida el trabajo de Dougherty (1992), quien es una de las primeras autoras en afirmar que el DNP es una de las prácticas concretas que sirven para renovar la empresa, por lo que entenderla permite un acercamiento a la cuestión de la renovación organizativa. En el desarrollo de un nuevo producto se produce un verdadero proceso de creación y explotación de cuatro tipos de conocimiento: el relativo a la función del producto, aquel que tiene que ver con la factibilidad de llevarlo a cabo, el que concierne al grado en el que se ajusta a las capacidades de la empresa, y el que se refiere a las nuevas tendencias del entorno, respectivamente. La habilidad de desarrollar adecuadamente cada uno de estos procesos de creación de conocimiento puede ser considerada como una verdadera capacidad central de una organización renovadora.

En las mismas fechas, otra autora, LeonardBarton (1992) insiste en dejar de considerar los proyectos de DNP como proyectos de rango medio, para empezar a valorar sus interrelaciones con el desarrollo de capacidades y con la estrategia. Aunque un proyecto determinado no altere profundamente las capaci- dades, prepara el camino para el cambio, al poner de manifiesto rigideces y permitir la incorporación de nuevas capacidades. Como vemos, sin utilizar aún el término de capacidades dinámicas, la autora se está refiriendo a este concepto. Sin embargo, los proyectos de desarrollo de nuevos productos y procesos son áreas de conflicto entre la necesidad de innovar y la retención de capacidades importantes. Se da la paradoja de que las capacidades centrales pueden, simultáneamente, favorecer e impedir la evolución.

La innovación de productos es un motor de renovación organizativa por varias razones, pero la más obvia es que la empresa puede utilizarlo para desarrollar nuevas habilidades, sistemas y conocimientos (Bowen et ál., 1994). Es más, una empresa puede utilizar conscientemente los proyectos de desarrollo de productos como medio de cambio para construir nuevas capacidades, e incluso puede considerar el aprendizaje como el objetivo último del proyecto. En otras palabras, la capacidad resultante puede ser - y a menudo es - más importante que el producto en sí mismo. La organización debería auditar cada proyecto de DNP para determinar qué se ha aprendido y establecer sistemas para aplicarlo a otros proyectos, logrando la renovación de las capacidades organizativas.

Las organizaciones maduras pueden renovarse mediante la búsqueda de un nuevo producto que reemplace, o al menos complemente, la actividad tradicional (Ruiz, 1998). La evolución y refinamiento de las competencias centrales, junto con la adquisición de recursos y competencias complementarios, son los elementos clave de las estrategias de 
renovación organizativa. Un método para la renovación continua de las competencias centrales, que permite incrementar la velocidad de cambio y superar la inercia, consiste en mover competencias periféricas hasta el núcleo y reforzarlas mediante la adquisición de competencias complementarias.

Para Karim y Mitchell (2000) el cambio de producto es, con toda claridad, una reconfiguración, un cambio en los recursos y capacidades de la organización. Eisenhardt y Martin (2000), en su trabajo dirigido a clarificar la noción de capacidades dinámicas, incluyen el desarrollo de productos entre ellas. En efecto, tratando de superar concepciones más bien vagas y tautológicas de lo que son capacidades dinámicas, identifican éstas con determinados procesos estratégicos y organizativos mediante los cuales los directivos alteran la base de recursos para generar nuevas estrategias creadoras de valor. El DNP, al igual que la toma de decisiones estratégicas o las alianzas, constituye uno de tales procesos, particularmente importante en mercados de gran dinamismo en los que es difícil mantener la concepción de la empresa como conjunto de recursos, puesto que éstos son añadidos, recombinados y retirados con regularidad.

Dicho esto, varias de las aportaciones en relación con las capacidades dinámicas pueden aplicarse al desarrollo de productos en particular. Así, el proceso variará en función del dinamismo del mercado. En mercados moderadamente dinámicos, será un proceso detallado, analítico, estable, con resultados predecibles, cercano a la concepción más tradicional de rutinas. En contraposición, en mercados altamente dinámicos, será un proceso más simple, altamente experimental, frágil y con resultados impredecibles. Además, el proceso de desarrollo de productos será idiosincrásico en sus detalles, pero mostrará características comunes entre empresas, debido a que existen formas más o menos efectivas de ejecutar capacidades particulares o "mejores prácticas".

Helfat y Raubitschek (2000) expresan la idea de que el conocimiento, las capacidades organizativas y los productos coevolucionan a lo largo del tiempo. En su modelo de la secuencia de productos explican cómo el conocimiento proporciona a la organización la base de la matriz de direcciones de desarrollo futuro. En otras palabras, la actual cartera de productos sirve como plataforma para las secuencias futuras. La estrecha relación entre productos y capacidades subyace en todo este trabajo, de tal manera que si bien la secuencia de productos se construye sobre la base de conocimientos y capacidades de la empresa, a la vez contribuye a aumentarla.

Uno de los autores que con más rotundidad han defendido el papel del desarrollo de productos en la renovación de las capacidades es sin duda Danneels, quien en su trabajo de 2002 afirma que el DNP es uno de los principales medios de renovación corporativa porque no sólo se sirve de, sino que también sirve para desarrollar las competencias de la empresa, permitiendo a ésta entrar en nuevos dominios de actividad. En efecto, al desarrollar un nuevo producto, la empresa puede disponer ya de las competencias (tecnológicas y de mercado) necesarias para ello, o puede construir tales competencias, lo que nos lle- 
vará a diferenciar entre las distintas formas de llevar a cabo la renovación de productos. En todo caso, el desarrollo y comercialización de nuevos productos son actividades que permiten expandir la base de competencias de la empresa, lo cual la capacita para seguir desarrollando otros nuevos productos.

Danneels denomina a las competencias tecnológicas y de mercado, competencias de primer orden, en cuanto que suponen la habilidad de realizar una tarea particular, y considera competencia de segundo orden a la habilidad para construir e incorporar en la empresa nuevas competencias de primer orden. Si bien esta es una terminología diferente a la utilizada por otros autores, podemos observar que coinciden básicamente en el contenido. El propio autor reconoce que se apoya en las capacidades de orden superior o "metacapacidades" de Collis (1994), quien definió a éstas como capacidades de aprender a aprender. El paralelismo con la clasificación de las capacidades en operativas y dinámicas también resulta evidente.

Algunas compañías pueden poseer excelentes competencias de primer orden u operativas (por ejemplo, conocen muy bien a sus clientes o dominan profundamente sus tecnologías), pero pueden fracasar cuando se enfrentan con el desafío del cambio si fallan las competencias de segundo orden. Estas últimas incluyen tareas como la investigación de mercados o la identificación de nuevas tecnologías prometedoras, las cuales podemos considerar que son claros ejemplos de la labor de monitorización y detección de estímulos del entorno para el consiguiente aprovechamiento de las oportunidades.
Verona y Ravasi (2003) también reconocen que los nuevos productos han sido señalados en numerosas ocasiones como la más natural fuerza promotora del cambio y la renovación a nivel corporativo. Es principalmente a través del proceso de desarrollo de productos como las organizaciones realizan su crítico rol de integrar conocimiento de diferente naturaleza (sobre todo científico, tecnológico y comercial), de una forma innovadora, y así generan nuevo conocimiento.

La planificación de la cartera de productos y el DNP son procesos que proporcionan las regularidades estructurales necesarias para lograr la adaptación de la eficiencia operativa, si bien los agentes deben esforzarse por innovar dentro de estas estructuras y lograr que faciliten la adaptación, mientras se mantienen conscientes de las trampas que pueden poner en peligro dicha adaptación (Gavetti, 2005).

Salvato (2009) intenta contribuir al concepto de capacidades dinámicas sugiriendo que tales capacidades pueden generarse a partir del refinamiento gradual de capacidades de más bajo nivel. Con este objetivo, el proceso concreto que investiga no es otro que el proceso de DNP. Así, encuentra que pequeñas actividades cotidianas realizadas por individuos de dentro y fuera de la organización y de todos los niveles jerárquicos, desempeñan un papel central en la formación de la capacidad de DNP y en su adaptación dinámica a lo largo del tiempo. Pero la intención de los directivos también desempeña un importante papel, por lo que la renovación organizativa puede ser en parte alcanzada mediante oportunas intervenciones directivas dirigidas a incor- 
porar experimentos exitosos en capacidades organizativas de nivel superior. En esta línea, algunos autores han empezado a incorporar el concepto de capacidades directivas dinámicas (Adner y Helfat, 2003).

El trabajo de Cetindamar et ál. (2009) puede considerarse un ejemplo reciente de investigación construida sobre la noción, ya bien asentada, de capacidad dinámica como habilidad de construir, integrar o reconfigurar capacidades operativas. Si bien es cierto que los autores analizan la dirección de la tecnología y no el DNP en su totalidad, sus conclusiones pueden extrapolarse al conjunto del proceso. Considerando como capacidades operativas las capacidades tecnológicas de la organización, se identifican las actividades/procesos/ rutinas que subyacen en tales capacidades, y se construye un modelo que explica cómo la dirección de la tecnología desarrolla y explota las capacidades tecnológicas sobre una base continua, actuando por tanto como una verdadera capacidad dinámica.

Newey y Zahra (2009) también subrayan la utilidad de operacionalizar las capacidades dinámicas y operativas a través de los procesos de planificación de la cartera de productos y desarrollo de productos, respectivamente. Es cierto que en este trabajo, consistente con Winter (2003), los autores califican a la capacidad de gestionar la cartera de productos como capacidad dinámica y a la capacidad de DNP como capacidad operativa. Es otra forma de operacionalizar la realidad, pero consideramos que el desarrollo de productos tiene rasgos suficientes como para ser considerado en sí mismo una capacidad dinámica $\mathrm{y}$, en todo caso, aun considerando las dos capacidades de diferente nivel, la segunda constituye la parte más relevante de la primera.

\section{Cuadro 1. Aportaciones sobre el papel del DNP en la renovación organizativa}

\begin{tabular}{|c|c|}
\hline Dougherty (1992) & $\begin{array}{l}\text { Es un proceso de creación y explo- } \\
\text { tación de conocimiento que sirve } \\
\text { para renovar la empresa. }\end{array}$ \\
\hline $\begin{array}{l}\text { Leonard-Barton } \\
\text { (1992) }\end{array}$ & $\begin{array}{l}\text { Prepara el camino para el cambio, } \\
\text { al poner de manifiesto rigideces y } \\
\text { permitir la introducción de nuevas } \\
\text { capacidades. }\end{array}$ \\
\hline $\begin{array}{l}\text { Bowen et ál. } \\
\text { (1994) }\end{array}$ & $\begin{array}{l}\text { Motor de renovación organizativa } \\
\text { porque la empresa puede utilizar- } \\
\text { lo para desarrollar nuevas habili- } \\
\text { dades, sistemas y conocimientos. }\end{array}$ \\
\hline Ruiz (1998) & $\begin{array}{l}\text { Nuevos productos que reempla- } \\
\text { cen o al menos complementen la } \\
\text { actividad tradicional, permiten la } \\
\text { renovación organizativa. }\end{array}$ \\
\hline $\begin{array}{l}\text { Karim y Mitchell } \\
(2000)\end{array}$ & $\begin{array}{l}\text { Supone una reconfiguración de } \\
\text { los recursos y capacidades de la } \\
\text { organización. }\end{array}$ \\
\hline $\begin{array}{l}\text { Eisenhardt y Mar- } \\
\text { tin }(2000)\end{array}$ & $\begin{array}{l}\text { Uno de los procesos que consti- } \\
\text { tuyen capacidades dinámicas, el } \\
\text { cual sirve para renovar y reconfigu- } \\
\text { rar los recursos de la organización } \\
\text { y contribuye a su renovación. }\end{array}$ \\
\hline $\begin{array}{l}\text { Helfat y Raubits- } \\
\text { chek (2000) }\end{array}$ & $\begin{array}{l}\text { El conocimiento, las capacida- } \\
\text { des organizativas y los produc- } \\
\text { tos coevolucionan a lo largo del } \\
\text { tiempo. }\end{array}$ \\
\hline Danneels (2002) & $\begin{array}{l}\text { Uno de los principales medios de } \\
\text { renovación corporativa porque no } \\
\text { sólo se sirve de, sino que también } \\
\text { sirve para desarrollar las compe- } \\
\text { tencias de la empresa. }\end{array}$ \\
\hline $\begin{array}{l}\text { Verona y Ravasi } \\
(2003)\end{array}$ & $\begin{array}{l}\text { La más natural fuerza promotora } \\
\text { del cambio y la renovación a nivel } \\
\text { corporativo. }\end{array}$ \\
\hline Gavetti (2005) & $\begin{array}{l}\text { Proceso que proporciona las regu- } \\
\text { laridades estructurales necesarias } \\
\text { para lograr la adaptación de la efi- } \\
\text { ciencia operativa. }\end{array}$ \\
\hline
\end{tabular}




\begin{tabular}{|l|l|}
\hline Salvato (2009) & $\begin{array}{l}\text { Capacidad dinámica que puede } \\
\text { generarse a partir del refinamien- } \\
\text { to gradual de capacidades de más } \\
\text { bajo nivel. }\end{array}$ \\
\hline $\begin{array}{l}\text { Cetindamar et ál. } \\
(2009)\end{array}$ & $\begin{array}{l}\text { Dirección de la tecnología como } \\
\text { capacidad dinámica que permite } \\
\text { desarrollar y explotar las capaci- } \\
\text { dades tecnológicas. }\end{array}$ \\
\hline $\begin{array}{l}\text { Newey y Zahra } \\
(2009)\end{array}$ & $\begin{array}{l}\text { Permite hacer operativas las ca- } \\
\text { pacidades dinámicas, ayudando a } \\
\text { clarificar cómo desarrollan las em- } \\
\text { presas su capacidad adaptativa. }\end{array}$ \\
\hline $\begin{array}{l}\text { Parente et ál. } \\
(2011)\end{array}$ & $\begin{array}{l}\text { Verdadero prototipo de capacidad } \\
\text { dinámica, favorecida por determi- } \\
\text { nadas técnicas de la dirección de } \\
\text { operaciones. }\end{array}$ \\
\hline
\end{tabular}

Fuente: elaboración propia

Newey y Zahra (2009) señalan que las capacidades operativas orientan las reconfiguraciones que las capacidades dinámicas deben emprender, por lo que se debería revisar la literatura que enfatiza cómo las capacidades dinámicas afectan a las operativas, pero ignora el efecto inverso de esta relación. En último término, estos autores consideran que la unión entre la literatura estratégica y la de desarrollo de productos contribuirá a superar la confusión acerca de la naturaleza de las capacidades dinámicas y ayudará a clarificar cómo las empresas desarrollan en la práctica su capacidad adaptativa.

Para terminar, entre los trabajos más recientes citaremos a Parente et ál. (2011), puesto que dichos autores empiezan por calificar al DNP como verdadero prototipo de capacidad dinámica. A partir de ahí estudian cómo dos estrategias actuales de la dirección de operaciones, la producción modular y la integración de proveedores, influyen en la habilidad de crear e introducir nuevos productos, y encuentran que, efectivamente, ejercen una influencia positiva, moderada por factores culturales.

Si bien podríamos citar un número mayor de trabajos, consideramos que los hasta aquí presentados constituyen una buena muestra de cómo se ha ido asentando en la literatura en los últimos años la idea de que el DNP reúne rasgos suficientes como para ser considerado una capacidad dinámica. Reconocemos además que los trabajos revisados destacan el papel reconfigurador de las capacidades operativas, dejando al descubierto el paralelismo entre el DNP y la concepción de capacidades dinámicas, en su formulación más extendida. No obstante, esta revisión vincula el proceso de DNP con un proceso de valoración más práctico que reflexivo, esto es, más cercano a los resultados de un análisis relativo a cómo mejorar las capacidades con que cuenta la empresa que a un proceso de reflexión extraordinario que se cuestiona cómo responder a los requisitos cambiantes de entornos inciertos y turbulentos.

\section{Opciones de desarrollo de nuevos productos y de reconfiguración de capacidades}

Una vez reconocida la caracterización en la literatura del DNP como una capacidad dinámica, hemos de señalar que sobre ésta nos proporciona una amplia variedad de tipologías. Entre ellas destacaremos las que se proponen desde la investigación relativa al DNP y desde la perspectiva del aprendizaje organizativo (March, 1991), seguida posteriormente por Danneels (2002). Para terminar el epígrafe, señalaremos algunos de los 
interrogantes suscitados en relación con estas cuestiones.

En la literatura sobre desarrollo de productos, a la hora de precisar qué significa la innovación de un producto, coexisten muchas clasificaciones diferentes: continuo/ discontinuo; evolucionado/revolucionario; incremental/radical; mayor/menor... (Danneels y Kleinschmidt, 2001), lo que dificulta los avances en el estudio de los procesos de DNP. Aun cuando se delimite el campo a lo técnico-productivo, el término puede evocar cambios de muy distinta naturaleza y con muy diversos grados de novedad (López, 2006). Tras revisar la literatura en profundidad, García y Calantone (2002) señalan que, pese a las divergencias, existe consenso en cuanto a considerar que el grado de novedad de un producto alude a discontinuidad en los aspectos comerciales o tecnológicos. Algunas escalas con medidas tecnológicas y comerciales para valorar el grado de novedad del nuevo producto pueden verse en García y Calantone (2002, p. 126), y también en Danneels y Kleinschmidt (2001, p. 366), aunque una de las propuestas más exhaustivas es la de Cooper y Kleinschmidt (1986).

También es cada vez mayor el consenso en cuanto a que el grado de novedad del producto es un concepto multidimensional (Danneels y Kleinschmidt, 2001, p. 369). Desde la perspectiva de la empresa, el grado de innovación presenta dos dimensiones: familiaridad (cercanía de los nuevos productos a los actuales en términos de mercados a los que se dirige o tecnologías que utiliza) y ajuste de los nuevos productos a los recursos y capacidades con los que cuenta la empresa. Según cuál sea la dimensión observada, los resultados de la investigación pueden ser diferentes. En el contexto de este trabajo, nos parece lo más apropiado medir el grado de novedad en términos de si los recursos y capacidades necesarios para desarrollar el nuevo producto son o no poseídos por la empresa. Como veremos seguidamente, esta perspectiva coincide con la distinción explotación-exploración de March (1991), y es utilizada después en el trabajo de Danneels (2002).

Junto con las aportaciones provenientes de la literatura sobre innovación, debemos señalar a continuación una de las más extendidas formas de clasificar los procesos de DNP, que es, en realidad, una forma de clasificar los procesos de aprendizaje organizativo. Se trata de la distinción entre estrategias de exploración y explotación, generalizada a partir del trabajo seminal de March (1991).

La estrategia de explotación incluye cuestiones capturadas por términos como "refinamiento, elección, producción, eficiencia, selección, implementación o ejecución" (March, 1991, p. 71). Su esencia es el refinamiento y la extensión de las competencias, tecnologías y paradigmas existentes. Sus resultados son positivos, próximos y predecibles. La estrategia de exploración implica conceptos como "búsqueda, variación, asunción de riesgos, experimentación, juego, flexibilidad, descubrimiento, innovación". Su esencia es, también, la experimentación con nuevas alternativas. Sus resultados son inciertos, distantes, a menudo negativos. $\mathrm{La}$ distancia en tiempo y espacio entre el aprendizaje y la consecución de resultados es generalmente mayor en el caso de la exploración, 
al igual que su incertidumbre (March, 1991, p. 71,85$) \mathrm{y}$, por tanto, representa un grado de innovación o de cambio mucho mayor que la explotación.

En el contexto del DNP la exploración incluye la búsqueda de nuevas informaciones de tecnología e información de mercado para la organización (Atuahene-Gima y Murray, 2007). Tal búsqueda no se realiza en un espacio bien definido, sino que expone a la empresa a ámbitos heterogéneos y a conocimiento alejado de su experiencia actual. En contraste, la explotación incluye la búsqueda de información en un espacio producto/ mercado bien definido, cercanamente relacionado con la experiencia previa de la empresa, buscando un conocimiento más profundo de su particular área de tecnología y mercado para mejorar la eficiencia e implementación.

Presentadas a nivel conceptual las estrategias de explotación y exploración, debemos reconocer que, a pesar de las notables diferencias entre ambas, su definición y sus implicaciones han estado rodeadas de una cierta ambigüedad y su operacionalización no ha estado exenta de dificultades. De entrada, puede ser necesario clarificar si ambas estrategias se distinguen por las diferencias en el tipo de aprendizaje seguido, o si se diferencian por representar la presencia versus la ausencia de aprendizaje (Gupta et ál., 2006). Según la lógica de March (1991), ambas estrategias incluyen aprendizaje porque incluso al replicar acciones pasadas, se acumula experiencia y se desciende en la curva de aprendizaje, aunque sea de una forma incremental.
Esfuerzos por superar los problemas operativos los encontramos en Holmqvist (2004) o en Uotila et ál. (2009), quienes definen el concepto de cantidad relativa de exploración, o también en Atuahene-Gima y Murray (2007), que proponen escalas de varios ítems para medir ambos constructos.

Una de las aportaciones que, debido en parte a la claridad de sus argumentos teóricos, facilita en buena medida el camino para posteriores desarrollos operativos, es la de Danneels (2002, 2008). En ésta nos detenemos a continuación. Un antecedente de dicha propuesta lo encontramos en la matriz de transición para medir la innovación de Abernathy y Clark (1985). Partiendo de que el DNP requiere la combinación de dos tipos de capacidades operativas, las capacidades tecnológicas y las de mercado, el autor considera que la empresa realiza explotación cuando trata de obtener un nuevo producto buscando nuevas combinaciones de sus capacidades tecnológicas y comerciales actuales, es decir, hace un aprendizaje que implica el uso de recursos o capacidades ya poseídos (dependencia del pasado). En el otro extremo, hace exploración si combina nuevas capacidades tanto tecnológicas como comerciales, es decir, lleva a cabo un aprendizaje que provoca la adición de nuevos recursos o capacidades (ruptura).

Es posible que la empresa siga una explotación o una exploración puras, aunque también puede que adopte opciones intermedias, buscando el apalancamiento de competencias, para conseguir la extracción de valor adicional de ciertos recursos infrautilizados. Las dos opciones intermedias buscan, o bien 
combinar las actuales capacidades tecnológicas con nuevas capacidades comerciales (apalancamiento de las capacidades tecnológicas), o bien combinar las actuales capacidades comerciales con nuevas capacidades tecnológicas (apalancamiento de las capacidades de mercado). El apalancamiento de las competencias trata de abstraer la competencia del producto particular en el que actualmente se materializa $\mathrm{y}$, a continuación, vincularla con una nueva competencia para desarrollar un nuevo producto. Se parte de la idea de que los recursos pueden ser definidos con independencia de su uso; son "piezas de un rompecabezas", las cuales "combina y recombina la empresa" (Penrose, 1959, pp. 25 y 70), por lo que se debe evitar una visión de la empresa centrada en el producto, y examinar más bien las capacidades en las que éste se basa (Hamel y Prahalad, 1994).

Recogiendo las aportaciones de Danneels, y tomando como referencia las capacidades operativas de las que parte la empresa, se puede señalar la existencia de cuatro grandes formas de llevar a cabo el DNP (cuadro 2).

La tipología de Danneels puede ponerse en relación con algunas de las más recientes aportaciones al enfoque de capacidades dinámicas, anteriormente revisadas. Así, tomando en consideración el tipo de capacidades implicadas en cada una de las opciones de desarrollo de producto, es posible relacionar dicha tipología con los distintos mecanismos de reconfiguración de capacidades propuestos por Lavie (2006). Podemos apreciar la existencia de un cierto paralelismo entre la explotación y el mecanismo de evolución de capacidades, la exploración y el mecanismo de sustitución, y también claramente encontramos analogías entre el apalancamiento y el mecanismo de transformación de capacidades. Con respecto a este último, el autor señalaba que "la configuración posterior al cambio se basa en una combinación de conocimiento y habilidades existentes con nuevos conocimientos y habilidades" (Lavie, 2006, p. 159), lo que lo convierte en un mecanismo de reconfiguración intermedio, y como tal, puede entenderse como un medio más rápido y menos arriesgado de lograr el crecimiento y renovación de la empresa (Hamel y Prahalad, 1994).

\section{Cuadro 2. Tipología de DNP}

\begin{tabular}{|c|c|c|c|}
\hline \multirow{2}{*}{\multicolumn{2}{|c|}{ Capacidades }} & \multicolumn{2}{|c|}{ Tecnológicas } \\
\hline & & Actuales & Nuevas \\
\hline \multirow{2}{*}{ 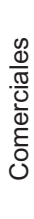 } & Actuales & Explotación & $\begin{array}{l}\text { Apalancamiento } \\
\text { capacidades co- } \\
\text { merciales }\end{array}$ \\
\hline & Nuevas & $\begin{array}{l}\text { Apalancamiento } \\
\text { capacidades tec- } \\
\text { nológicas }\end{array}$ & Exploración \\
\hline
\end{tabular}

Fuente: Danneels (2002, p. 1105)

Por otro lado, la tipología de Danneels (2002) también puede ser conectada con el trabajo de Teece (2007), quien destacaba que bajo las capacidades dinámicas subyacen tres tipos de procesos: monitorización del entorno, aprovechamiento de oportunidades y reconfiguración. Ellonen et ál. (2009) encuentran que las empresas que poseen fuertes capacidades dinámicas en las tres áreas consiguen apalancar sus capacidades. En contraste, las compañías con débiles o con un solo tipo de capacidades dinámicas (monitorización, principalmente) producen innovaciones más radicales, las cuales requieren la adición de nuevas capaci- 
dades tanto tecnológicas como comerciales. En particular, las firmas que no poseen fuertes capacidades del último tipo (reconfiguración) son incapaces de aprovechar sus actuales recursos y capacidades cuando innovan.

Hechas estas precisiones conceptuales y operativas en relación con las alternativas de explotación y exploración, pasamos a plantear brevemente algunos de los interrogantes que, desde su aparición, han rodeado a estos términos y que tienen que ver con cuestiones como: ¿son ambas opciones compatibles?, ¿cuál de ellas es seguida con más frecuencia por las empresas?, ¿cuál lleva a los mejores resultados?

Los primeros interrogantes se suscitan ante la afirmación de March (1991) de que la interacción entre ambas ocurre en forma de juego de suma cero, donde exploración y explotación compiten por recursos escasos, atención y rutinas organizativas; por consiguiente, deben ser vistas como dos extremos de un continuo, en el que más cantidad de recursos destinados a una significa menos recursos destinados a la otra.

Esta afirmación ha sido revisada con posterioridad por otros autores (Katila y Ahuja, 2002; Nerkar, 2003), quienes han observado que en ocasiones ambas alternativas funcionan como ortogonales, en el sentido de que más cantidad de una no significa menos cantidad de la otra. A la vista de estos resultados, podemos afirmar que no existen argumentos universales a favor de la continuidad o de la ortogonalidad (Gupta et ál., 2006), sino que la relación entre exploración y explotación depende en buena medida de si ambas com- piten por recursos escasos y de cuál sea la unidad de análisis (individuo, organización, proyecto de DNP).

Si bien March (1991) entiende explotación y exploración como extremos de un continuo, en ningún caso quiere decir con ello que la empresa deba decantarse en exclusiva por una u otra opción, puesto que la dedicación exclusiva a una de ellas puede acarrear graves problemas. Por esta razón, mantener un adecuado balance entre exploración y explotación resulta primordial para asegurar la supervivencia y prosperidad de la organización. A partir del trabajo de March, los beneficios que conlleva el mantenimiento de este balance han sido puestos de manifiesto por un buen número de investigadores en sus trabajos, incrementándose con ello, cada vez más, el interés por analizar esta cuestión, lo que ha dado lugar a la aparición de un nuevo tópico en la literatura: la ambidestreza (Raisch et ál., 2009).

En cualquier caso, reconociendo que para las empresas no es fácil mantener el balance, surge la cuestión de cuál es, de entre las dos, la alternativa por la que principalmente se decantan. March (1991) reconoce que existe una clara tendencia a la explotación, influida en buena medida por los sistemas de reparto de costes y beneficios en la organización, así como por la competencia interorganizativa. Los resultados de la explotación son generalmente más ciertos que los de la exploración, por lo que ésta queda relegada a un segundo término (Levinthal y March, 1993).

En este sentido, encontramos una coincidencia con los hallazgos del enfoque de capaci- 
dades dinámicas, el cual enfatiza que las empresas, en el desarrollo de sus competencias, siguen una senda de dependencia respecto de las elecciones pasadas lo que puede impedir la apertura a nuevas posibilidades. Esto resulta peligroso en situaciones en las que el cambio es necesario, en las cuales es probable que el proceso de adaptación a través de la explotación sea efectivo a corto plazo, pero autodestructivo a largo plazo (March, 1991). Esta cuestión nos conduce de nuevo al debate de capacidades dinámicas versus capacidades organizativas, incluido su proceso de dinamización (Schreyogg y Kliesch-Eberl, 2007; Dávila, 2012). Así, hablar del DNP como capacidad dinámica supondría atribuirle las limitaciones que ésta comporta, al utilizar patrones rutinarios ya establecidos en el desarrollo de nuevas soluciones. Esto resulta especialmente evidente en el caso de la explotación, en tanto que el DNP descansa en las capacidades tecnológicas y comerciales ya disponibles. Asimismo, en el caso de DNP con apalancamiento, tanto tecnológico como comercial, se reconoce de forma explícita una dependencia con respecto al pasado y a la presencia de procesos de DNP ya preestructurados y guiados por las capacidades disponibles. Todos estos casos responderían bien, por tanto, a la noción de capacidades dinámicas, pero no se ajustarían a procesos de dinamización abiertos y flexibles. En cambio, el proceso de exploración se presenta como un proceso de DNP alejado de las capacidades disponibles y, en consecuencia, con mayor potencial para reflejar la función de dinamización de las capacidades organizativas. Si es entendido como una capacidad dinámica, el proceso de exploración estará en buena medida guiado por esquemas preesta- blecidos y sujeto a la flexibilidad que marca el proceso rutinario de renovación. De esta forma, si entendemos el DNP con exploración como proceso de dinamización de capacidades organizativas, podremos vincularlo con un proceso más abierto, que se propone desde una mirada alejada del proceso operativo y se enfoca hacia los retos de la dinámica del contexto competitivo.

\section{Conclusiones}

Este trabajo nace con el propósito de revisar la investigación del proceso de DNP a la luz de las propuestas de las capacidades dinámicas. Con ese fin hemos comenzado por atender al concepto de capacidades dinámicas, incluyendo también los aspectos más controvertidos que al respecto recoge la literatura. A continuación revisamos la caracterización del DNP y las distintas tipologías propuestas en la literatura. Así, resulta fácil comprobar cómo frecuentemente se reconoce el papel del DNP como elemento clave en el desarrollo y reconfiguración de las capacidades operativas. Por ello, con frecuencia es calificado como una capacidad dinámica vinculada al proceso de renovación de la empresa.

La noción de capacidad dinámica es un concepto en evolución, que en las concepciones más recientes abarca desde la capacidad de identificar y aprovechar oportunidades hasta la reconfiguración de la base de recursos y capacidades de la empresa. Ahora bien, este concepto no está exento de críticas que, lejos de resolverse, abren nuevos horizontes en la investigación. Tal es el caso del carácter tautológico del concepto y la disyuntiva que se plantea con respecto a la coherencia entre 
el propio concepto de capacidad dinámica, su base en procesos rutinarios y el carácter dinámico que se le atribuye. La propuesta de separación entre el concepto original de capacidades organizativas y su proceso externo de dinamización plantea un proceso más abierto, que quizás se ajusta mejor a la concepción del proceso de renovación organizativa que reclama la dinámica del entorno.

De otro lado, la literatura sobre DNP reconoce la diversidad de procesos que comporta la innovación de productos y, por ello, alberga distintas tipologías. Sin duda, una de las más conocidas es la que distingue explotación de exploración. La primera se relaciona con procesos de DNP vinculados total o parcialmente con capacidades existentes. Así, se pueden caracterizar como procesos de reconfiguración del catálogo de capacidades, delimitados y anticipados por las propias capacidades de la empresa. Los procesos de DNP vinculados a la exploración aluden en cambio a procesos de reconfiguración de capacidades no dependientes de las capacidades previas. Ahora bien, interpretados como capacidades dinámicas, resultarán igualmente, si bien con menor intensidad, procesos de innovación condicionados y anticipados en cierta medida por las actuales capacidades de la empresa. Sin embargo, enfocados desde la óptica de las capacidades organizativas y su proceso de dinamización, los procesos de exploración adquieren una dimensión más abierta y novedosa que permite una reconfiguración inspirada en los retos que emanan del entorno, y no tanto en la mejora práctica de la situación actual. Nos encontramos entonces frente a un mecanismo de verdadera renovación organizativa y no de parciales mejoras en las prácti- cas organizativas. Nuestro trabajo ofrece, por tanto, una visión sobre el proceso de DNP y las concepciones en las cuales este proceso contribuye a la renovación.

\section{Referencias}

Abernathy, W. J. y Clark, K. B. (1985). Innovation: Mapping the winds of creative destruction. Research Policy, 14 (1), 3-22.

Adner, R. y Helfat, C. E. (2003). Corporate effects and dynamic managerial capabilities. Strategic Management Journal, 24 (10), 1011-1025.

Amit, R. y Schoemaker, P. (1993). Strategic assets and organizational rent. Strategic Management Journal, 14 (1), 33-46.

Atuahene-Gima, K. y Murray, J. (2007). Exploratory and exploitative learning in new product development: A social capital perspective on new technology ventures in China. Journal of International Marketing, 15 (2), 1-29.

Barreto, I. (2010). Dynamic capabilities: A review of past research and an agenda for the future. Journal of Management, 36 (1), 256-280.

Bowen, K., Clark, K. B., Holloway, C. A. y Wheelwright, S. (1994). Development projects: The engine of renewal. Harvard Business Review, Sept-October, 110-120.

Brown, S. L. y Eisenhardt, K. (1995). Product development: Past research, present findings and future directions. Academy of Management Review, 20 (2), 343-378.

Cepeda, G. y Vera, D. (2007). Dynamic capabilities and operational capabilities: A knowledge management perspective. Journal of Business Research, 60, 426-437.

Cetindamar, D., Phaal, R. y Probert, D. (2009). Understanding technology management as a dynamic capability: A framework for technology management activities. Technovation, 29, 237-246. 
Collis, D. J. (1994). How valuable are organizational capabilities? Strategic Management Journal, Winter Special Issue 15, 143-152.

Cooper, R. G. (1994). Perspective: Third generation new product processes. Journal of Product Innovation Management, 11 (1), 3-14.

Cooper, R. G. y Kleinschmidt, E. J. (1986). An investigation into the new product process: Steps, deficiencies and impact. Journal of Product Innovation Management, 3 (2), 71-85.

Danneels, E. (2002). The dynamics of product innovation and firm competences. Strategic Management Journal, 23 (12), 1095-1121.

Danneels, E. (2008). Organizational antecedents of second-order competences. Strategic Management Journal, 29 (5), 519-543.

Danneels, E. y Kleinschmidt, E. J. (2001). Product innovativeness from the firm's perspective: Its dimensions and their relation with project selection and performance. Journal of Product Innovation Management, 18 (6), 357-373.

Dávila, J. C. (2012). La doble dimensión de una capacidad organizacional: evidencias de una organización sin ánimo de lucro que compite en el mercado. Cuadernos de Administración, 25 (44), 11-37.

Dougherty, D. (1992). A practice centered model of organizational renewal through product innovation. Strategic Management Journal, 13, 77-92.

Eisenhardt, K. M. y Martin, J. A. (2000). Dynamic capabilities: What are they? Strategic Management Journal, October-November Special Issue 21, 1105-1121.

Ellonen, H. K, Wikström, P. y Jantunen, A. (2009). Linking dynamic-capability portfolios and innovation outcomes. Technovation, 29, 753-762.

García, R. y Calantone, R. (2002). A critical look at technological innovation typology and innovativeness terminology: A literature review.
Journal of Product Innovation Management, 19 (2), 110-132.

Gavetti, G. (2005). Cognition and hierarchy: Rethinking the microfoundations of capabilities development. Organization Science, 16, 599-617.

Gomes, J., De Weerd-Nederhof, P., Pearson, A. y Cunha, M. (2003). Is more always better? An exploration of the differential effects of functional integration on performance in new product development. Technovation, 23, 185-191.

Gupta, A. K., Smith, K. G. y Shalley, C. E. (2006). The interplay between exploration and exploitation. Academy of Management Journal, 49 (4), 693-706.

Hamel, G. y Prahalad, C. K. (1994). Competing for the future. Breakthrough strategies for seizing control of your industry and creating the markets of tomorrow. Boston: Harvard Business School Press.

Helfat, C. y Raubitschek, R. (2000). Product sequencing: Co-evolution of knowledge, capabilities and products. Strategic Management Journal, 21, 961-979.

Helfat, C. E. y Peteraf, M. A. (2003). The dynamic resource- based view: Capability lifecycles. Strategic Management Journal, October Special Issue 24, 997-1010.

Helfat, C. E., Finkelstein, S., Mitchell, W., Peteraf, M., Singh, H., Teece, D. y Winter, S. G. (2007). Dynamic capabilities: Understanding strategic change in organizations. Oxford: Blackwell.

Henderson, R. H. y Cockburn, I. C. (1994). Measuring competence: Exploring firm effects in pharmaceutical research. Strategic Management Journal, Winter Special Issue 15, 63-84.

Holmqvist, M. (2004). Experiential learning processes of exploitation and exploration within and between organizations: An empirical study of product development. Organization Science, 15 (1), 70-81. 
Karim, S. y Mitchell, W. (2000). Path-dependent and path-breaking change: Reconfiguring business resources following acquisitions in the U.S. medical sector (1978-1995). Strategic Management Journal, 21, 1061-1081.

Katila, R. y Ahuja, G. (2002). Something old, something new: A longitudinal study of search behavior and new product introduction. Academy of Management Journal, 45 (6), 1183-1194.

Kogut, B. y Zander, U. (1992). Knowledge of the firm, combinative capabilities, and the replication of technology. Organization Science, 3 (3), 383-397.

Lavie, D. (2006). Capability reconfiguration: An analysis of incumbent responses to technological change. Academy of Management Review, 31 (1), 153-174.

Leonard-Barton, D. (1992). Core capabilities and core rigidities: A paradox in managing new product development. Strategic Management Journal, Summer Special Issue 13, 111-125.

Levinthal, D. A. y March, J. G. (1993). The myopia of learning. Strategic Management Journal, Winter Special Issue 14, 95-112.

López, G. A. (2006). Perspectivas para el análisis de la innovación: un recorrido por la teoría. Cuadernos de Administración, 19 (31), 243-273.

Makadok, R. (2001). Toward a synthesis of the resource-based and dynamic-capability views of rent creation. Strategic Management Journal, 22 (5), 387-401.

March, J. G. (1991). Exploration and exploitation in organizational learning. Organizational Science, 2 (1), 71-87.

Montoya-Weiss, M. M. y Calantone, R. (1994). Determinants of new product performance: A review and meta-analysis. Journal of Product Innovation Management, 11, 397-417.
Nerkar, A. (2003). Old is gold? The value of temporal exploration in the creation of new knowledge. Management Science, 49 (2), 211-229.

Newey, L. R. y Zahra, S. A. (2009). The evolving firm: How dynamic and operating capabilities interact to enable entrepreneurship. British Journal of Management, 20, 81-100.

Nonaka, I. y Takeuchi, H. (1995). The knowledgecreating company: How Japanese companies create the dynamics of innovation. New YorkOxford: Oxford University Press.

Parente, R. C., Baack, D. W. y Hahn, E. D. (2011). The effect of supply chain integration, modular production, and cultural distance on new product development: A dynamic capabilities approach. Journal of International Management, 17 (4), 278-290.

Penrose, E. T. (1959). The theory of the growth of the firm. New York: John Wiley \& Sons.

Perks, H. (2004). Exploring processes of resource exchange and co-creation in strategic partnering for new product development. International Journal of Innovation Management, 8 (1), 37-61.

Priem, R. L. y Butler, J. E. (2001). Is the resourcebased 'view' a useful perspective for strategic management research? Academy of Management Review, 26 (1), 22-40.

Raisch, S., Birkinshaw, J., Probst, G. y Tushman, M. L. (2009). Organizational ambidexterity: Balancing exploitation and exploration for sustained performance. Organization Science, 20 (4), 685-695.

Ruiz, J. (1998). Turnaround and renewal in a Spanish shipyard. Long Range Planning, 31 (1), 51-59.

Salvato, C. (2009). Capabilities unveiled: The role of ordinary activities in evolution of product development processes. Organization Science, 20 (2), 384-409. 
Schreyögg, G. y Kliesch-Eberl, M. (2007). How dynamic can organizational capabilities be? Towards a dual-process model of capability dynamization. Strategic Management Journal, 28 (9), 913-933.

Teece, D. J. (2007). Explicating dynamic capabilities: The nature and microfoundations of (sustainable) enterprise performance. Strategic Management Journal, 28 (13), 1319-1350.

Teece, D. J. (2009). Dynamic capabilities and strategic management: organizing for innovation and growth. Oxford University Press.

Teece, D. J., Pisano, G. y Shuen, A. (1997). Dynamic capabilities and strategic management. Strategic Management Journal, 18 (7), 509-533.

Uotila, J., Maula, M., Keil, T. y Zahra, S. A. (2009). Exploration, exploitation, and financial performance: analysis of S\&P 500 corporations. Strategic Management Journal, 30 (2), 221-231.

Varela, J. y Benito, L. (2005). New product development process in Spanish firms: Typology, antecedents and technical/ marketing activities. Technovation, 25, 395- 405.

Verona, G. y Ravasi, D. (2003). Unbundling dynamic capabilities: An exploratory study of continuous product innovation. Industrial and Corporate Change, 12 (3), 577-606.

Winter, S. G. (2003). Understanding dynamic capabilities. Strategic Management Journal, October Special Issue 24, 991-995.

Zahra, S. A., Sapienza, H. J. y Davidsson, P. (2006). Entrepreneurship and dynamic capabilities: A review, model and research agenda. Journal of Management Studies, 43 (4), 917-955.

Zollo, M. y Winter, S. G. (2002). Deliberate learning and the evolution of dynamic capabilities. Organization Science, 13 (3), 339-351.

Zott, C. (2003). Dynamic capabilities and the emergence of intraindustry differential firm performance: Insights from a simulation study. Strategic Management Journal, 24 (2), 97-125. 
